\title{
Fresnel Correction of the Beckmann Model
}

\author{
Hossein Ragheb ${ }^{1,2}$ and Edwin R. Hancock ${ }^{1}$ \\ ${ }^{1}$ Department of Computer Science, University of York, York, UK. \\ ${ }^{2}$ Faculty of Engineering, Bu-Ali Sina University, Hamedan, Iran. \\ ${ }^{1}$ \{hossein, erh\}@cs.york.ac.uk ${ }^{2}$ ragheb@basu.ac.ir
}

\begin{abstract}
In this paper we suggest a modification to the Beckmann-Kirchhoff model of rough surface reflectance that can be used for surfaces of intermediate roughness. One of the problems with the existing Beckmann model is that it fails for large angles of incidence. This problem was overcome for rough surfaces by Vernold and Harvey who use a Lambertian form factor. Here we take this work one step further by using a Fresnel coefficient to account for subsurface reflectance attenuation. This study also aims to show the existing modelling gaps between different variants of the Beckmann model.
\end{abstract}

\section{Introduction}

The modelling of rough surface reflectance is important in both computer vision and computer graphics, and has been the subject of sustained research activity for some four decades. In fact the quest for a reflectance model that can accurately account for the observed surface radiance under a variety of roughness conditions, and a variety of viewing geometries has proved to be an elusive one. Surface roughness can be characterised in a number of ways. For very-rough surfaces, one approach is to use a model which describes the distribution of surface wall cavity angles [5]. For slightly-rough surfaces, i.e. ones that present a shiny appearance, roughness can be modelled using the angular distribution of microfacets [8]. An alternative that can capture both effects is to describe the roughness phenomenon using the variance and the correlation length of variations in the surface height distribution $[11,3]$.

In computer vision there have been a number of recent attempts to model rough surface reflectance in a phenomenological way. These models attempt to account for roughness as departures from Lambert's cosine law. For instance, Oren and Nayar [5] use the surface cavity picture to model very-rough surfaces. The departures from Lambert's law are greatest at large light scattering angles. However, this model has been criticised due to the unrealistic nature of its isotropy assumption by van Ginneken et al. [11] who have recently developed a model that can be used to predict reflectance from isotropic rough surfaces. The parameters of their model are the surface roughness measured in terms of the RMS slope, the albedo, and the balance between the diffuse and specular components. For slightly-rough or shiny surfaces, Wolff [9], on the other hand, has a model motivated by physics. Here refractive attenuation in the surface-air layer is modelled by multiplying Lambert's cosine law by two Fresnel terms. One of these accounts for light incidence and the second for reflection. For medium roughness, Wolff, Nayar and Oren [10] have combined the two methodologies by assuming that reflection from each microfacet in the Oren-Nayar model follows the Fresnel model which is used in the Wolff model.

However, it is interesting that some of the earliest work in the area was undertaken by Beckmann [1], who used the Kirchhoff integral to model the wave scattering of light 
from rough surfaces characterised using the variance and correlation length of the surface relief distribution. The model is mathematically quite complex, and is hence not well suited for analysis tasks of the type encountered in computer vision. In particular, it is not a simple modification of Lambert's law. However, He et al. [3] exploited an improved representation of the Kirchhoff integral for surface synthesis in computer graphics. They proposed a comprehensive model that incorporates complex factors including surface statistics, sub-layer scattering, and polarization. Also, Stam [7] has recently applied the Kirchhoff approach to include diffraction effects in surface rendering. Unfortunately, the Beckmann model fails to account for the observed radiance at large scatter angles due to energy absorbtion, self shadowing and multiple scattering effects. Some of the problems have recently been overcome by Vernold and Harvey [12] who have used a simple Lambertian form factor to modify Beckmann's predictions. Moreover, the B-K model provides closed-form solutions only for slightly-rough and very-rough surfaces.

The aim in this paper is to report a new variant of the Beckmann model that can be applied to surfaces of intermediate roughness. Our idea is to extend the model to account for the subsurface refractive attenuation of light prior to wave scattering. This effect can be accommodated by multiplying the Kirchhoff scattering kernel by a form factor that depends on the Fresnel reflecton coefficient. In this study, we use a correction term, similar to that used by Wolff [9], in place of the Lambertian term factor of Vernold and Harvey. This model has a number of advantages. first, it allows the Beckmann model to be used for conditions of intermediate roughness. Second, it provides a good fit to reflectance measurements.

\section{Beckmann-Kirchhoff scatter theory}

The Beckmann-Kirchhoff (B-K) theory attempts to account for the wave interactions of light with rough surfaces. The starting point is Kirchhoff's integral which gives the scattered light-field amplitude. Beckmann's contribution was to show how to apply the theory to rough surfaces, and how to obtain simplifications to the Kirchhoff integral under different roughness conditions. The details of the theory are comprehensively described in the monographs of Beckmann and Spizzichino [1], and by Ogilvy [4]. By requiring that the surface has both a Gaussian height distribution and a Gaussian correlation function, according to the B-K theory the total scattered intensity is given by

$$
I\left(\theta_{i}, \theta_{r}, \phi_{r}\right)=I_{0} e^{-g}+\frac{\pi T^{2} F^{2}\left(\theta_{i}, \theta_{r}, \phi_{r}\right) e^{-g}}{A} \sum_{n=1}^{\infty}\left[\frac{g^{n}}{n ! n} \exp \left(\frac{-v_{x y}^{2} T^{2}}{4 n}\right)\right]
$$

The incident beam has zenith angle $\theta_{i}$ and azimuth angle $\phi_{i}=\pi$, and the reflected beam has zenith angle $\theta_{r}$ and azimuth angle $\phi_{r}$ (on local tangent planes). From trigonometry it follows that $v_{x}=k\left(\sin \theta_{i}-\sin \theta_{r} \cos \phi_{r}\right), v_{y}=-k\left(\sin \theta_{r} \sin \phi_{r}\right), v_{z}=-k\left(\cos \theta_{i}+\right.$ $\left.\cos \theta_{r}\right), v_{x y}^{2}=v_{x}^{2}+v_{y}^{2}, g=\sigma^{2} v_{z}^{2}$ and $k=2 \pi / \lambda$, where $\lambda$ is the wavelength. The parameter $\sigma$ is the root-mean-square (RMS) height deviation of the topographic surface features about the mean surface level. The height values are generally measured at equally spaced digitized data points. The correlation length $T$ is defined as the lag-length at which the Gaussian correlation function drops to $1 / e$ of its maximum. The geometrical factor is

$$
F\left(\theta_{i}, \theta_{r}, \phi_{r}\right)=\left(1+\cos \theta_{i} \cos \theta_{r}-\sin \theta_{i} \sin \theta_{r} \cos \phi_{r}\right) / \cos \theta_{i}\left(\cos \theta_{i}+\cos \theta_{r}\right)
$$

The quantity $g$ has been used in the literature to divide surfaces into three broad categories. These are a) slightly-rough $(g \ll 1)$, b) moderately-rough $(g \approx 1)$ and c) veryrough $(g \gg 1)$ surfaces. The parameter $A$ is the area of a plane sheet on which the scat- 
tering coefficient $I_{0}$ is defined after integration of the mean scattered field equation [1]. The first term in Eq. (1) determines the specular component. The scattering coefficient $I_{0}$ vanishes everywhere except near the direction of specular reflection. The second term, i.e. the infinite series, determines the diffuse component. The number of terms that need to be used in the summation depends on the roughness with respect to the wavelength. When we consider the specular direction $\left(\theta_{i}=\theta_{r}\right.$ and $\left.\phi_{r}=0\right)$, then both $v_{x}$ and $v_{y}$ vanish and $F=1$. Hence the optical intensity is proportional to $\exp (-g)$. We have recently shown [6] how to use this simple equation to estimate the roughness for slightly-rough surfaces.

The first interesting case arises when the surface is slightly rough $(g \ll 1)$, and so the series in Eq. (1) converges rapidly. In practice, only the first term needs to be considered and the diffuse intensity becomes

$$
I_{d}\left(\theta_{i}, \theta_{r}, \phi_{r}\right) \approx\left(\pi g T^{2} F^{2} / A\right) \exp \left[-\left(g+T^{2} v_{x y}^{2} / 4\right)\right]
$$

The second interesting case arises when the surface is very rough $(g \gg 1)$ when compared to the test wavelength. Under these conditions the specular term becomes insignificant and the total (or diffuse) intensity is

$$
I\left(\theta_{i}, \theta_{r}, \phi_{r}\right) \approx\left(\pi F^{2} T^{2} / A v_{z}^{2} \sigma^{2}\right) \exp \left(-v_{x y}^{2} T^{2} / 4 v_{z}^{2} \sigma^{2}\right)
$$

We also simplify the model for the case when the angle between the light-source direction $\vec{L}$ and the viewing direction $\vec{V}$ is small, i.e. $\vec{L} \approx \vec{V}$, and so $\theta_{i}=\theta_{r}=\theta$ and $\phi_{r}=\pi$. In this case, the B-K model for very-rough surfaces (Eq. 4) reduces to

$$
I(\theta) \approx\left(T^{2} \lambda^{2} / 16 \pi A \sigma^{2} \cos ^{6} \theta\right) \exp \left(-T^{2} \tan ^{2} \theta / 4 \sigma^{2}\right)
$$
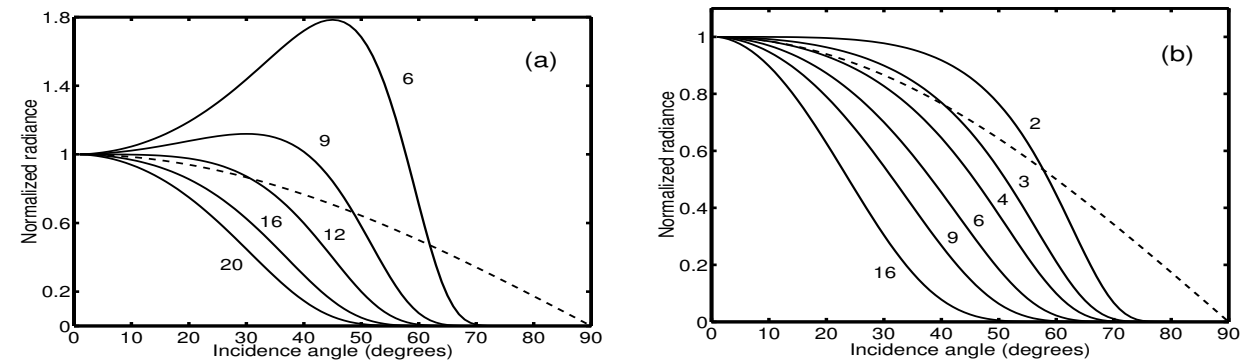

Figure 1: Normalized radiance versus $\theta_{i}$ using (a) the original (Eq. 5) and (b) the modified (Eq. 6) B-K model for several values of $\eta=(T / \sigma)^{2}$; Lambert's law (dashed).

\subsection{Modified Beckmann-Kirchhoff model}

The failure of the B-K theory to handle wide-angle scattering and large angles of incidence has been highlighted by several authors [2, 3, 4]. To solve this problem, Vernold and Harvey [12] have recently replaced the geometrical factor $F^{2}$ used in the B-K model with a Lambertian term that depends only on the cosine of the incidence angle $\cos \theta_{i}$. This modification gives reasonable experimental agreement with scattering data for rough surfaces at large angles of incidence and large scattering angles. The modification relies on empirical and phenomenological arguments. However, this does not diminish its pragmatic use since it includes some effects (corresponding to non-paraxial angles) which are not included in the original B-K model. Making this replacement, the Vernold-Harvey (V-H) modification to the B-K model for very-rough surfaces for the case of $\vec{L} \approx \vec{V}$ is

$$
I(\theta) \approx\left(T^{2} \lambda^{2} / 16 \pi A \sigma^{2} \cos \theta\right) \exp \left(-T^{2} \tan ^{2} \theta / 4 \sigma^{2}\right)
$$

Hence, the $1 / \cos ^{6} \theta$ behavior of the original B-K model (Eq. 5) is replaced by a slower $1 / \cos \theta$ behavior. Fig. 1 illustrates the effect of this different behavior for veryrough surfaces. The different curves in each panel show the normalized radiance as a 
function of the incidence angle for different values of $\eta=(T / \sigma)^{2}$. The dashed curve in each panel is the prediction of Lambert's cosine law. Panel (a) is for the original B-K model (Eq. 5), while panel (b) is for the modified B-K model of Vernold-Harvey (Eq. 6). Panel (a) demonstrates that the original B-K model diverges significantly from the Lambertian curve for large angles of incidence. Here, the normalized radiance peaks at large scatter angles for small values of $\eta$ due to the $1 / \cos ^{6} \theta$ term. Panel (b), on the other hand, shows that the $\mathrm{V}-\mathrm{H}$ modification to the B-K model does not exhibit these problems. From the different curves it is also clear that the larger the surface roughness $\sigma$ with respect to correlation length $T$, the higher the normalized radiance. In particular, for $\eta=2$, the brightening effects are relatively moderate compared to the Lambertian curve.

\section{Phenomenological reflectance models}

In this section we review the models of Oren and Nayar [5] and Wolff [9] briefly.

\subsection{Oren-Nayar model for rough surfaces}

Oren and Nayar have developed a diffuse reflectance model for rough surfaces [5]. They have used the roughness model proposed by Torrance and Sparrow [8] that assumes the surface is composed of extended symmetric V-shaped cavities. Each cavity consists of two planar facets. The width of each facet is assumed to be small compared to its length. The roughness of the surface is specified using a probability distribution function for the facet slopes. Finally, each facet is assumed to follow the Lambertian model. Here we focus on their qualitative model in which interreflections are ignored. According to this model, for a point on a rough surface with a roughness parameter $\sigma_{\alpha}$, illuminant incidence direction $\left(\theta_{i}, \phi_{i}\right)$ and reflectance direction $\left(\theta_{r}, \phi_{r}\right)$, the surface radiance is

$$
L_{r}\left(\theta_{i}, \theta_{r}, \Delta \phi ; \sigma_{\alpha}\right)=\left(\rho E_{0} / \pi\right) \cos \left(\theta_{i}\right)\{A+B \max [0, \cos (\Delta \phi)] \sin (\alpha) \tan (\beta)\}
$$

The parameters $A$ and $B$ (which are dimensionless) are only dependent on the surface roughness $\sigma_{\alpha}$ (which is measured in degrees or radians). Specifically $A=1.0-$ $0.5 \sigma_{\alpha}^{2} /\left(\sigma_{\alpha}^{2}+0.33\right)$ and $B=0.45 \sigma_{\alpha}^{2} /\left(\sigma_{\alpha}^{2}+0.09\right)$. Also, $\Delta \phi=\phi_{r}-\phi_{i}, \alpha=\max \left[\theta_{i}, \theta_{r}\right]$ and $\beta=\min \left[\theta_{i}, \theta_{r}\right]$. It is important to note that the model reduces to the Lambertian case when $\sigma_{\alpha}=0$. Finally, the parameter $\rho$ represents the surface albedo (reflectivity) which is assumed to be constant. Physical significance of the Oren-Nayar model becomes clearer if we simplify to the case where $\vec{L} \approx \vec{V}$ (i.e. $\theta_{r}=\theta_{i}=\theta$ and $\Delta \phi=0$ ). Under such conditions we can approximate the $\mathrm{O}-\mathrm{N}$ model by making the substitutions $\cos (\Delta \phi)=1$ and $\alpha=\beta=\theta$. As a result, the simplified surface radiance (for $\rho=1)$ is

$$
L_{r}(\theta)=A \cos (\theta)+B \sin ^{2}(\theta)
$$

Hence, the correction to Lambert's law is additive and proportional to $\sin ^{2} \theta$. This term is greatest at the occluding boundary, and hence results in limb brightening. Fig. 2.a shows the radiance versus the incidence angle for different values of $\sigma_{\alpha}$ in Eq. (8). The effect of increasing the roughness is to make the radiance function flatter with incidence angle. Hence, the contrast between the limb and the remainder of the object is reduced.

\subsection{Wolff model for smooth surfaces}

Wolff has developed a physically motivated model for diffuse reflectance from smooth surfaces [9]. The model accounts for subsurface refraction using a Fresnel attenuation factor, which modifies a Lambertian radiance function in a multiplicative way. According to this model, the surface radiance is given by

$$
L_{r}\left(\theta_{i}, \theta_{r}, n\right)=\rho L_{i} \cos \left(\theta_{i}\right)\left[1-f\left(\theta_{i}, n\right)\right]\left\{1-f\left(\sin ^{-1}\left[\left(\sin \theta_{r}\right) / n\right], 1 / n\right)\right\}
$$


The attenuation factor, $0 \leq f\left(\alpha_{i}, n\right) \leq 1.0$, is governed by the Fresnel function

$$
f\left(\alpha_{i}, r\right)=\left[\sin ^{2}\left(\alpha_{i}-\alpha_{t}\right) / 2 \sin ^{2}\left(\alpha_{i}+\alpha_{t}\right)\right]\left[1+\cos ^{2}\left(\alpha_{i}+\alpha_{t}\right) / \cos ^{2}\left(\alpha_{i}-\alpha_{t}\right)\right]
$$

The transmission angle $\alpha_{t}$ of light into the dielectric surface is given by Snell's law:

$$
r=\left(\sin \alpha_{i}\right) /\left(\sin \alpha_{t}\right) \Rightarrow \quad \alpha_{t}=\sin ^{-1}\left[\left(\sin \alpha_{i}\right) / r\right]
$$

The parameter $n$ is the index of refraction of the dielectric medium. When light is transmitted from air into a dielectric $r=n$ and $\alpha_{i}=\theta_{i}$. However, when transmission is from a dielectric into air, then $r=1 / n$ and $\alpha_{i}=\sin ^{-1}\left[\left(\sin \theta_{r}\right) / n\right]$. The Wolff model deviates from the Lambertian form when the Fresnel terms become significant. Almost all commonly found dielectric materials have an index of refraction, $n$, in the range $[1.4,2.0]$. As a result the Fresnel function is weakly dependent upon the index of refraction for most dielectrics. The value of the scaling factor $\rho$ is very nearly constant over most incidence and reflectance angles [9]. We can also simplify the Wolff model to the case when $\vec{L} \approx \vec{V}$. When $\theta_{r}=\theta_{i}=\theta$ the two Fresnel terms are identical and the radiance simplifies to

$$
L_{r}(\theta, n)=\cos (\theta)[1-f(\theta, n)]^{2}
$$

The Fresnel term has the effect of depressing the radiance for near-normal incidence. In Fig. 2.b we plot the radiance versus incidence angle using the simplified radiance function (Eq. 12) for different values of $n$. The greater the index of refraction, the greater the reduction in the near-normal reflectance.
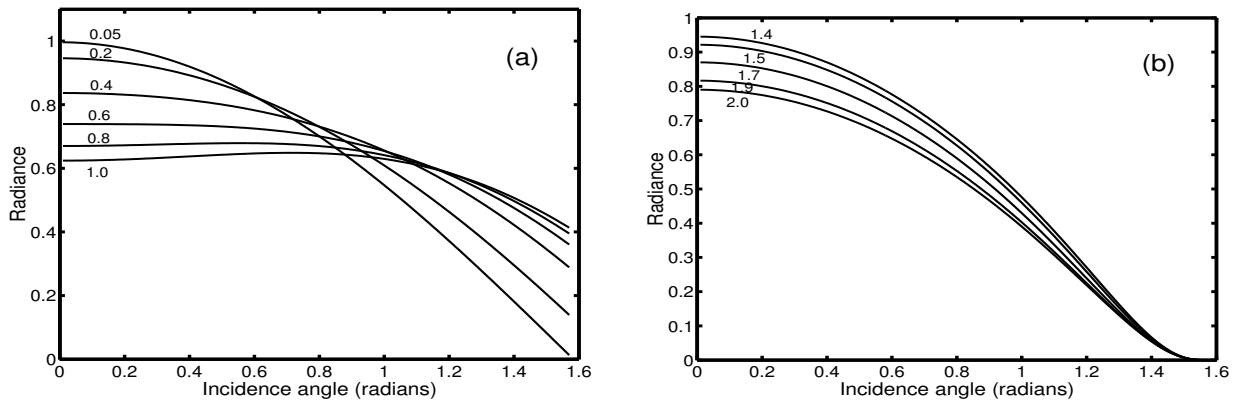

Figure 2: Radiance versus incidence angle (radians) using (a) the Oren-Nayar model (Eq. 8) for several values of $\sigma_{\alpha}$ (radians), and (b) the Wolff model (Eq. 12) for several values of $n$.

\section{Comparisons}

In this section, we compare the two variants of the B-K model described earlier with the Oren-Nayar (O-N) model and with the Wolff model (Fig. 3). We compare the O-N model with the modified B-K model for very-rough surfaces. However, it should be noted that the surface slope parameter in the B-K model $(\sigma / T)$ is a physics-based parameter and does not convey the same meaning as its counterpart, i.e. $\sigma_{\alpha}$, in the O-N model. There is no roughness parameter appearing in the Wolff model and the changes caused by varying the index of refraction $n$ are very small. Nonetheless, we compare this model with the modified B-K model that applies to slightly-rough surfaces. These comparisons are shown in Fig. 3 for the case of $\vec{L} \approx \vec{V}$. In both panels of the figure we plot the normalized radiance versus the incidence angle using different models. The dash-dot curve in each panel corresponds to the Lambert's cosine law. Fig. 3.a shows the curves for both the modified B-K model (Eq. 6, solid curve) and the O-N model (Eq. 8, dashed curve). Here, we use $\sigma / T=1.5$ and $\sigma_{\alpha}=20^{\circ}$. The values are chosen so that the shapes of the different distributions are roughly comparable. The main points to note from this plot are 
as follows. First, the O-N model and the modified B-K model are close in form for small angles. Second, the modified B-K model tends to zero as $\theta$ approaches $\pi / 2$, while the O-N model does not tend to zero due to the $B \sin ^{2} \theta$ term. This limb brightening effect is a consequence of the interdependence of $A$ and $B$, and only vanishes if $\sigma_{a}=0$. The modified B-K model, on the other hand gives zero radiance for $\theta_{i}>73^{\circ}$. Hence, the two models result in quite different behaviours at large angles of incidence.
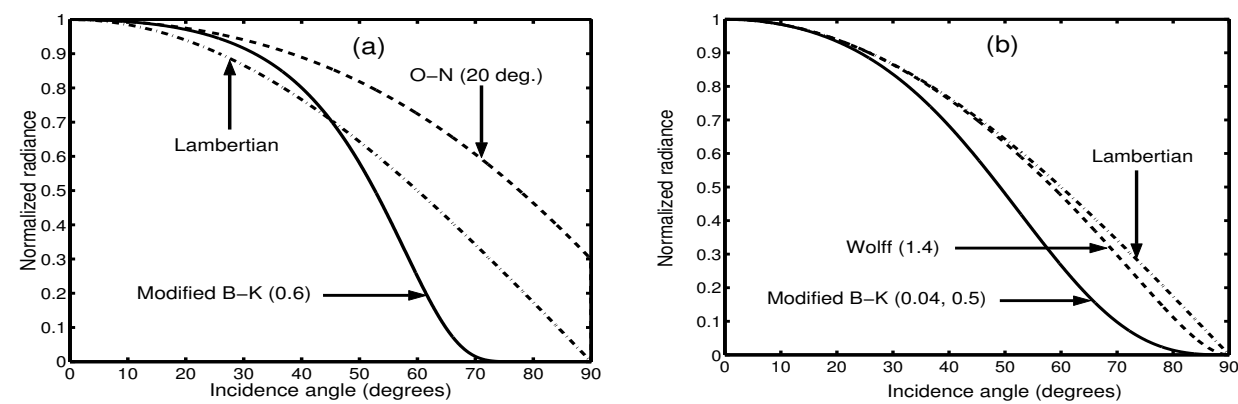

Figure 3: (a) Comparison of the modified B-K model (Eq. 6) with the Oren-Nayar model (Eq. 8) for $\sigma / T=0.6$ and $\sigma_{\alpha}=20^{\circ}$; (b) Comparison of the modified B-K model (Eq. 3) with the Wolff model (Eq. 12) for $\sigma=0.04 \mu \mathrm{m}, T=0.5 \mu \mathrm{m}, n=1.4$ and $\lambda=0.5 \mu \mathrm{m}$.

Since Wolff does not have an explicit roughness parameter in his model (Eq. 12), comparing the B-K model and the Wolff model is not straightforward. The reason for this is that although the shape of the radiance curves for the modified B-K model vary significantly as the roughness parameters $\sigma$ and $T$, and also the wavelength $\lambda$ are varied, in the case of the Wolff model the changes brought about by varying the index of refraction parameter are small. However, in Fig. 3.b we show the modified B-K model for slightlyrough surfaces (Eq. 3) using $\sigma=0.04 \mu \mathrm{m}, T=0.5 \mu \mathrm{m}$ and $\lambda=0.5 \mu \mathrm{m}$ in the same plot where we show the Wolff model using $n=1.4$. Despite considerable differences between the two models, in the next section we show how these models can be combined to bridge the existing gap between different variants of the modified B-K model.

\section{Fresnel correction}

It is well known that the B-K model cannot handle surface self-shadowing and multiple scattering [2]. In Section 2.1, we described how the $\mathrm{V}-\mathrm{H}$ modification improves the reflectance predictions of the B-K model for large incidence and scattering angles. However, the model is also limited to very-rough or slightly-rough surfaces. In this section, we examine the effect of combining the Fresnel coefficient and the B-K model to develop a model that is applicable to surfaces of intermediate roughness. Although the B-K model [1] provides closed-form equations for slightly-rough $(g \ll 1$, Eq. 3$)$ and very-rough $(g \gg 1$, Eq. 4) surfaces, this is not the case for moderately-rough surfaces $(g \approx 1)$.

The Fresnel coefficient has been widely used to account for subsurface scattering. For instance, Wolff [9] has used it to correct the Lambertian model for smooth surfaces. Torrance and Sparrow [8] have included the Fresnel coefficient in their specular intensity model. It is also used in the complex reflectance model of He et al. [3] which attempts to account for a number of effects including subsurface scattering. Our approach is similar to that followed by Wolff et al. [10] which combines the Fresnel term with the Oren-Nayar model to develop a model for moderately-rough surfaces. We exploit the Fresnel model used by Wolff (Eqs. 9-11) which includes the effects of both incidence and reflectance 
angles. Specifically, the geometrical term $F^{2}$ in the B-K model for very-rough surfaces (Eq. 4) is replaced by a Fresnel correction term to produce the corrected B-K model. Whereas Vernold and Harvey [12] have replaced the $F^{2}$ term by $\cos \left(\theta_{i}\right)$, we replace it by the factor $\cos ^{2}\left(\theta_{i}\right)$ (which compensates for the lack of energy conservation) multiplied by two Fresnel terms. The correction term is

$$
\mu\left(\theta_{i}, \theta_{r}, n\right)=\left[1-f\left(\theta_{i}, n\right)\right]\left\{1-f\left(\sin ^{-1}\left[\left(\sin \theta_{r}\right) / n\right], 1 / n\right)\right\} \cos ^{2}\left(\theta_{i}\right)
$$

The correction hence combines the advantages delivered by the V-H modification [12] with some additional ones too. The B-K model for very-rough surfaces (Eq. 4) is applicable as long as the condition $g \gg 1$ holds. However, it is not precisely clear for which values of $g$ this condition is violated. For slightly-rough surfaces, on the other hand, the condition for applicability of the B-K model (Eq. 3 ) is that $g \ll 1$. Again, there is no precise boundary for this condition to hold. In conclusion, the condition for switching from one equation to another is ambiguous and causes practical problems, specifically when the surfaces under study are of the medium or unknown roughness. This problem may be overcome if the Fresnel corrected form of the B-K model (corrected B-K model) is used instead over a wider range.
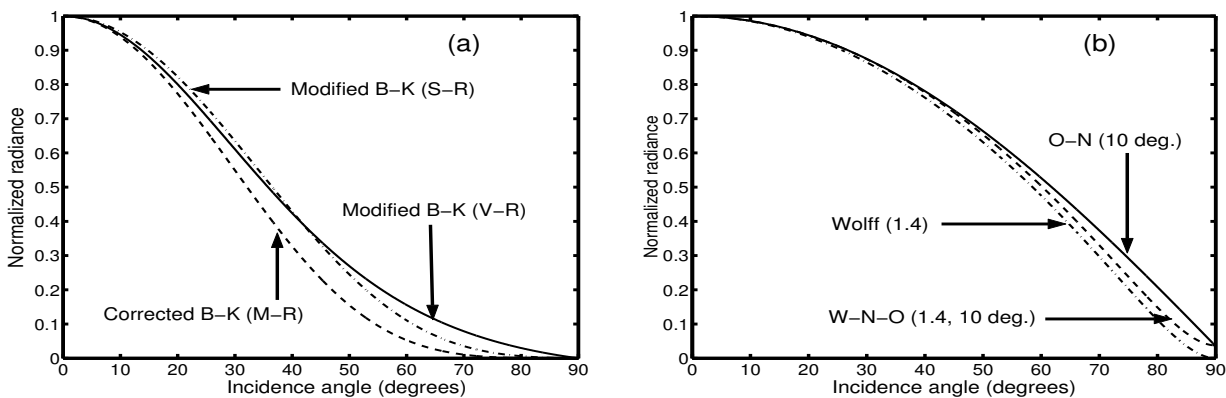

Figure 4: Normalized radiance versus $\theta_{i}$ using: (a) the modified B-K model for Very-Rough surfaces, the corrected B-K model for Moderately-Rough surfaces and the modified B-K model for Slightly-Rough surfaces for $\theta_{r}=0$ where $\sigma=0.05 \mu \mathrm{m}, T=0.25 \mu \mathrm{m}$ and $\lambda=0.7 \mu \mathrm{m}$; (b) the Oren-Nayar (O-N), Wolff and combined (W-N-O) models for $\vec{L} \approx \vec{V}$ where $\sigma_{\alpha}=10^{\circ}$ and $n=1.4$.

One of the problems caused by this ambiguity is that under conditions where $\theta_{r}=0$, and for values of RMS roughness $\sigma$ and correlation length $T$ where $0.1<g<0.5$, the scattered intensity predicted by the B-K model for slightly-rough surfaces may become higher than that for very-rough surfaces. This is a phenomenon which is supported neither by physical nor by geometrical optics. One example for this situation is when $\sigma=0.05 \mu \mathrm{m}$, $T=0.25 \mu \mathrm{m}$ and $\lambda=0.7 \mu \mathrm{m}$, which gives $g \leq 0.4$. Here it is not clear which of the conditions $g \ll 1$ or $g \approx 1$ is applicable. This problem is shown in Fig. 4.a. For this case, the scattered intensities predicted by the modified B-K model for both slightly-rough and very-rough surfaces for most incidence angles are not reliable. However, when the corrected B-K model is used, the predictions of scattered intensity for most incidence angles are reliable. We also show the combined model of Wolff et al. [10] in Fig. 4.b for the case of $\vec{L} \approx \vec{V}$. Here, for the Oren-Nayar model, we use $\sigma_{\alpha}=10^{\circ}$ which corresponds to a moderately-rough surface. For the Wolff model, the index of refraction is set to $n=1.4$. Finally, the combined model of Wolff-Nayar-Oren uses both $\sigma_{\alpha}=10^{\circ}$ and $n=1.4$. Both plots of Fig. 4 show how the Fresnel term can be combined with reflectance models for rough surfaces and also results in alternative models for moderately-rough surfaces. 
There may be other difficulties when deciding which variant of the B-K model applies to a surface. Firstly, the quantity $g=\left[(2 \pi \sigma / \lambda)\left(\cos \theta_{i}+\cos \theta_{r}\right)\right]^{2}$, which is used to distinguish between three categories of rough surfaces does not depend on the correlation length $T$. However, the parameter which controls the shape of the variants of the $\mathrm{B}-\mathrm{K}$ model for very-rough surfaces is not the RMS roughness $\sigma$ alone. Instead it is the slope parameter, i.e. $\sigma / T$. Hence, a value of $\sigma$ which gives a $g \gg 1$ may be associated with surfaces with different values of $T$ and $\sigma / T$. Secondly, in contrast to the case for slightly-rough surfaces, the shape of the scattered intensity curve for very-rough surfaces does not change by changing the wavelength $\lambda$. However, since $g$ is proportional to $1 / \lambda^{2}$, changing $\lambda$ may change the category into which the surface falls while $\sigma$ and $T$ are fixed.

To compare the models, we replace the geometrical term $F^{2}$ in the B-K model for very-rough surfaces (Eq. 4 ) once by our correction term, i.e. $\mu\left(\theta_{i}, \theta_{r}, n\right)$, and once by the V-H modification term, i.e. $\cos \theta_{i}$. In Fig. 5, the plots show the normalized radiance versus incidence angle for a typical RMS slope value $\sigma / T=0.6$. We perform the comparisons for two different illumination conditions. Panel (a) of the figure is for the case of $\vec{L} \approx \vec{V}$, i.e. $\theta_{i}=\theta_{r}$ and $\phi_{r}=\pi$, whereas panel (b) is for the case of $\theta_{r}=0$ and $0 \leq \theta_{i} \leq \pi / 2$.
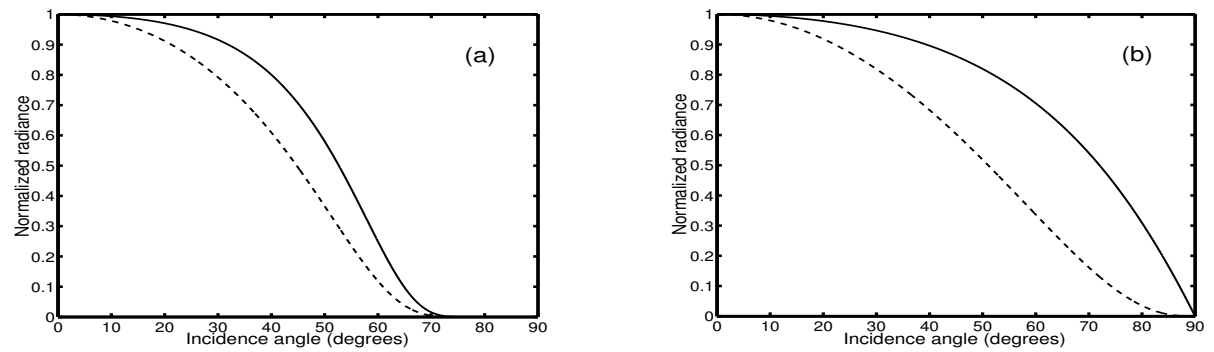

Figure 5: Normalized radiance versus $\theta_{i}$ for (a) $\theta_{r}=\theta_{i}$ and $\phi_{r}=\pi$, and for (b) $\theta_{r}=0$ and $0 \leq \theta_{i} \leq \pi / 2$, by applying the Vernold-Harvey modification (solid) and our Fresnel correction (dashed) terms to the B-K model of Eq. (4) where $\sigma / T=0.6$.

In both plots, the radiance curve corresponding to our correction term is lower than that corresponding to the V-H modification. However in panel (b) where $\theta_{r}=0$ the difference between the two curves is higher. The main reason for this is that the V-H modification term which is identical to the cosine Lambert's law does not depend on the reflectance angle. However, our correction term contains both a Fresnel term for incidence and a Fresnel term for reflectance angle. The effect of these Fresnel terms are different for different illumination conditions. When $\theta_{r}=0$, the Fresnel term corresponding to $\theta_{r}$ is fixed and only the Fresnel term corresponding to $\theta_{i}$ varies as $\theta_{i}$ varies. However, when $\theta_{i}=\theta_{r}=\theta$, the Fresnel terms are identical and both vary as $\theta$ varies. Again, in panel (a) where $\vec{L} \approx \vec{V}$, both variants of the B-K model fail for large angles of incidence and reflectance. However, this behaviour does not occur in panel (b) where $\theta_{r}=0$.

\section{Experimental results}

In this section we experiment with samples of sandpaper and wallpaper as moderatelyrough surfaces. These surfaces have been illuminated using a single collimated tungsten light-source with a parallel beam. However we used a blue filter which provides a single wavelength of $\lambda=0.4 \mu \mathrm{m}$. The light-source was placed far away from the objects and collimated using a cylinder. The camera was focused automatically while the aperture ratio was set manually. Both the viewing direction $\vec{V}$ (optical axis of the camera) and the 
light-source direction $\vec{L}$ are approximately aligned with the $z$ axis.

Under these conditions, we capture 18 images for each surface by rotating the surface from $\theta_{i}=0$ to $\theta_{i}=90^{\circ}$ with intervals of $5^{\circ}$. Next, we find a mean value for each image by averaging the grey-scale values in a window containing sufficient number of pixels. We show some of the images of sandpaper corresponding to $\theta_{i}=0,10^{\circ}, \ldots, 60^{\circ}$ in Fig. 6 . As $\theta_{i}$ increases the width of images become smaller until at $\theta_{i} \approx 90^{\circ}$ the grey-scale level drops to zero and no part of the surface samples are visible. Using the mean brightness values obtained from the real-world data we plot the normalized radiance versus incidence angle as shown in both panels of Fig. 7 by solid curves. To compare the data with different variants of the B-K model, we need to estimate the roughness parameters for each surface.
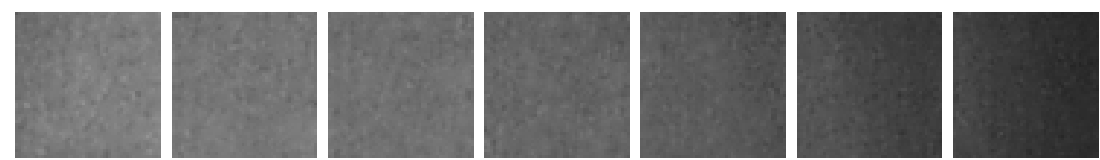

Figure 6: (a-g) Images of sandpaper samples for $\theta_{i}=0,10^{\circ}, 20^{\circ}, . ., 60^{\circ}$, respectively.
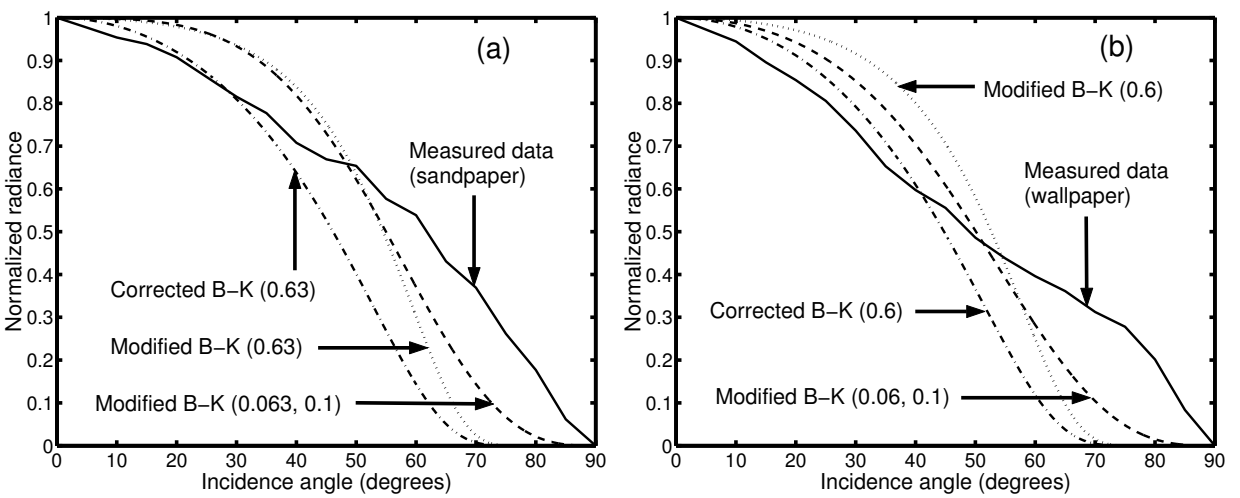

Figure 7: Normalized radiance versus $\theta_{i}(\vec{L} \approx \vec{V})$ using: real-world data for sandpaper and wallpaper, the corrected B-K model for moderately-rough surfaces, the Vernold-Harvey modification to the B-K model for slightly-rough, and, for very-rough surfaces.

Here we use our method described previously in [6] to estimate the surface slope $m=$ $\sigma / T$ for each surface. The method uses the modified B-K model for very-rough surfaces together with two images of each surface taken under off-normal illumination from two different incidence angles. The estimates are $m=0.63$ for the sandpaper and $m=0.6$ for the wallpaper. Since we can not estimate $\sigma$ and $T$ separately, we use $\sigma=0.063 \mu \mathrm{m}$ and $T=0.1 \mu \mathrm{m}$ for the sandpaper and $\sigma=0.06 \mu \mathrm{m}$ and $T=0.1 \mu \mathrm{m}$ for the wallpaper which give the best fit of the modified B-K model for slightly-rough surfaces to the data. We show these plots in panels (a) and (b) of Fig. 7 for the sandpaper and the wallpaper. In each plot, we show the modified B-K model for slightly-rough surfaces as a dashed curve, the modified B-K model for very-rough surfaces as a dotted curve, and the corrected B-K model for moderately-rough surfaces as a dash-dot curve.

There are a number of points to note from these plots. First, the data curve is closer to the corrected B-K model curve for small and intermediate angles. Second, when the modified B-K model for slightly-rough surfaces is used, the radiance curve for small and intermediate angles is higher than the curve corresponding to the modified B-K model for very-rough surfaces. For these incidence angles the condition $g \ll 1$ may be violated and 
switches to $g \approx 1$ or to $g \gg 1$. Finally, although the data curve for large incidence angles is closer to the modified B-K model for very-rough surfaces, the difference between the two curves is considerable. The problem may originate from the simplistic assumption in the B-K model that the surface correlation function is Gaussian. Some authors $[4,6]$ have shown that when the surface correlation function is exponential, the B-K model results in better agreement with real-world data.

\section{Conclusions}

In this paper we have suggested a correction term as an alternative to the modification of Vernold and Harvey [12] that can be used in conjunction with the B-K model. While the Vernold-Harvey approach is aimed at improving the B-K model for large incidence and scattering angles, we aim to provide a corrected variant of the B-K model for moderatelyrough surfaces. In doing this, we have exploited the Fresnel coefficient in a manner similar to that of Wolff et al. [10]. Our experiments show that the corrected model results in good agreement with real-world reflectance data for most incidence angles. Further, the applicability of the Fresnel correction term can be tested when the correlation function is assumed to be exponential. It would also be interesting to investigate whether an integrated correction process could be developed that would apply under all roughness conditions, hence avoiding the need to switch between different approximations.

\section{References}

[1] P. Beckmann and A. Spizzichino, The Scattering of Electromagnetic Waves from Rough Surfaces, Pergamon, New York, 1963.

[2] J.E. Harvey, C.L. Vernold, A. Krywonos and P.L. Thompson, "Diffracted Radiance: A Fundamental Quantity in a Non-paraxial Scalar Diffraction Theory," Applied Optics, vol. 38, no. 31, 1999, pp. 6469-6481.

[3] X.D. He, K.E. Torrance, F.X. Sillion and D.P. Greenberg, "A Comprehensive Physical Model for Light Reflection," ACM Computer Graphics, vol. 25, 1991, pp. 175-186.

[4] J.A. Ogilvy, Theory of Wave Scattering from Random Rough Surfaces, Adam Hilger, Bristol, 1991.

[5] M. Oren and S.K. Nayar, "Generalization of the Lambertian Model and Implications for Machine Vision,” Int'l J. Computer Vision, vol. 14, no. 3, 1995, pp. 227-251.

[6] H. Ragheb and E.R. Hancock, "Estimating Surface Characteristics using Physical Reflectance Models," Proc. IEEE CVPR, pp. 177-184 .

[7] J. Stam, "Diffraction Shaders," Computer Graphics, Proc. ACM SIGGRAPH 99, ACM Press, New York, 1999, pp. 101-110.

[8] K.E. Torrance and E.M. Sparrow, "Theory for Off-Specular Reflection from Roughened Surfaces," J. Optical Society of Amer., vol. 57, no. 9, 1967, pp. 1105-1114.

[9] L.B. Wolff "Diffuse Reflectance Model for Smooth Dielectric Surfaces," J. Optical Society of America A, vol. 11, no. 11, 1994, pp. 2956-2968.

[10] L.B. Wolff, S.K. Nayar and M. Oren, "Improved Diffuse Reflection Models for Computer Vision,” Int'l J. Computer Vision, vol. 30, no. 1, 1998, pp. 55-71.

[11] B. van Ginneken, M. Stavridi and J. Koenderink, "Diffuse and Specular Reflectance from Rough Surfaces," Applied Optics, vol. 37, no. 1, 1998, pp. 130-139.

[12] C.L. Vernold, and J.E. Harvey, "A Modified Beckmann-Kirchoff Scattering Theory for Non-paraxial Angles,"Proceedings of the SPIE, vol. 3426, 1998, pp. 51-56. 